\title{
AGGREGATE MORPHOLOGY AND AQUEOUS DISPERSIBILITY OF SPRAY-DRIED POWDERS OF CELLULOSE NANOCRYSTALS
}

\section{Supporting Information}

Yussef Esparza ${ }^{1}$, Tri-Dung $\mathrm{Ngo}^{2}$, Carole Fraschini ${ }^{3}$, Yaman Boluk ${ }^{1, *}$

${ }^{1}$ Department of Civil and Environmental Engineering, University of Alberta, Edmonton, Alberta, T6G 1H9, Canada

${ }^{2}$ Innotech Alberta, 250 Karl Clark Road, Edmonton, Alberta, T6N 1E4, Canada

${ }^{3}$ FPInnovations, 570 Boulevard Saint Jean, Pointe-Claire, Quebec, H9R 3J9, Canada 


\section{Methodology}

\section{Scanning electron microscopy}

The CNCs suspension was diluted to $0.01 \%$ and a drop was deposited on a 300 -mesh copper TEM grid. After one minute of waiting time, the excess of the liquid part was taken by adsorbing on a filter paper and a drop of uranyl acetate $2 \%$ was deposited on top of the stained sample. The excess of liquid was blotted again after one minute and the remaining stain was dried under a gentle flow of $\mathrm{N}_{2}$ gas. CNCs were imaged in the transmission mode (STEM) in a Hitachi S-4800 FESEM microscope (Hitachi, Japan) at an accelerating voltage of $30 \mathrm{kV}$.

\section{Isothermal titration calorimetry}

Interaction of OPE-100 surfactant molecules and CNCs was evaluated by isothermal titration calorimetry (ITC) experiments using a TA Nano ITC 2G instrument (TA, New Castle, DE, USA) at 25 and $70^{\circ} \mathrm{C}$. CNCs suspension of $1.7 \mathrm{wt} \%$ or deionized water is added to the sample cell (volume of $1015 \mu \mathrm{L}$ ). A solution of OPE-100 at $5 \mathrm{mM}$ concentration is loaded into the $100 \mu \mathrm{L}$ syringe of the instrument. An automatic titration of $5 \mu \mathrm{L}$ of OPE100 solution in every five minutes was performed in water and $\mathrm{CNC} 1.7 \%$. The heat released or adsorbed during the titration process was recorded by the instrument and analyzed using the NanoAnalyze software (TA, New Castle, DE, USA). The critical micellar concentration (cmc) was calculated as the peak value in a graph of the first derivative of $\Delta \mathrm{H}$ vs. surfactant concentration. 
Results

STEM images of CNC suspension

STEM images of $0.01 \%$ CNC suspension are shown in Figure S1.

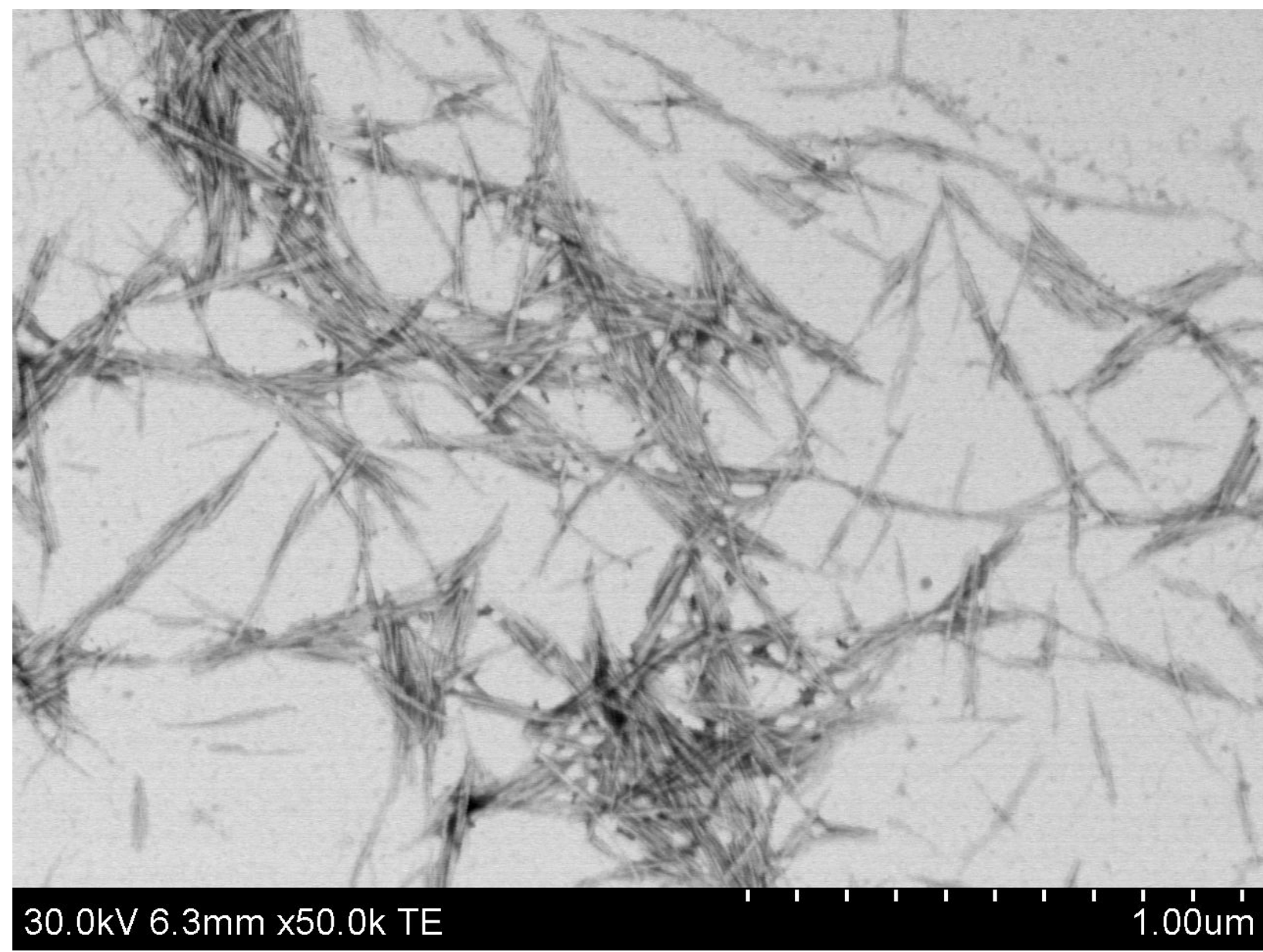




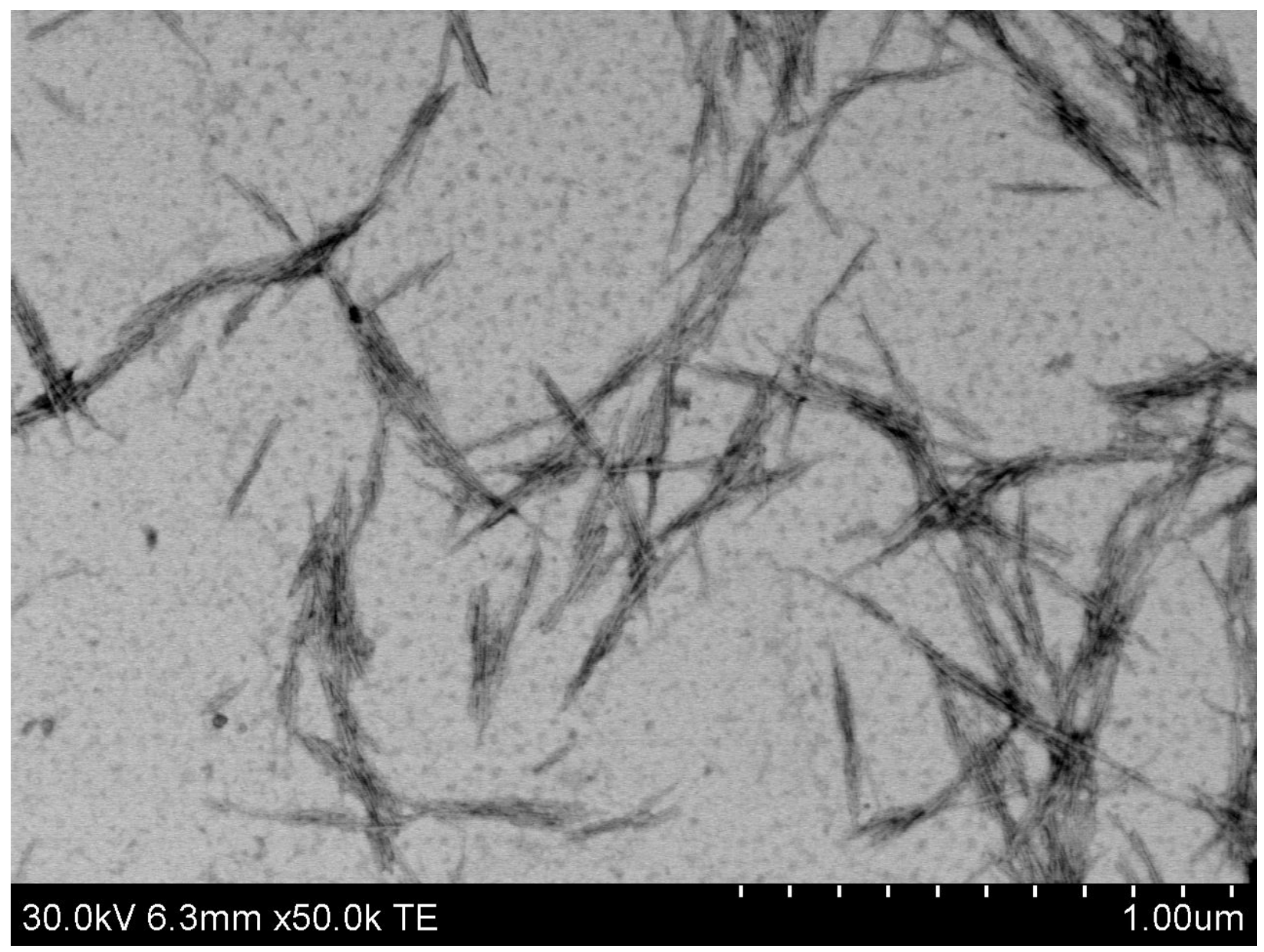




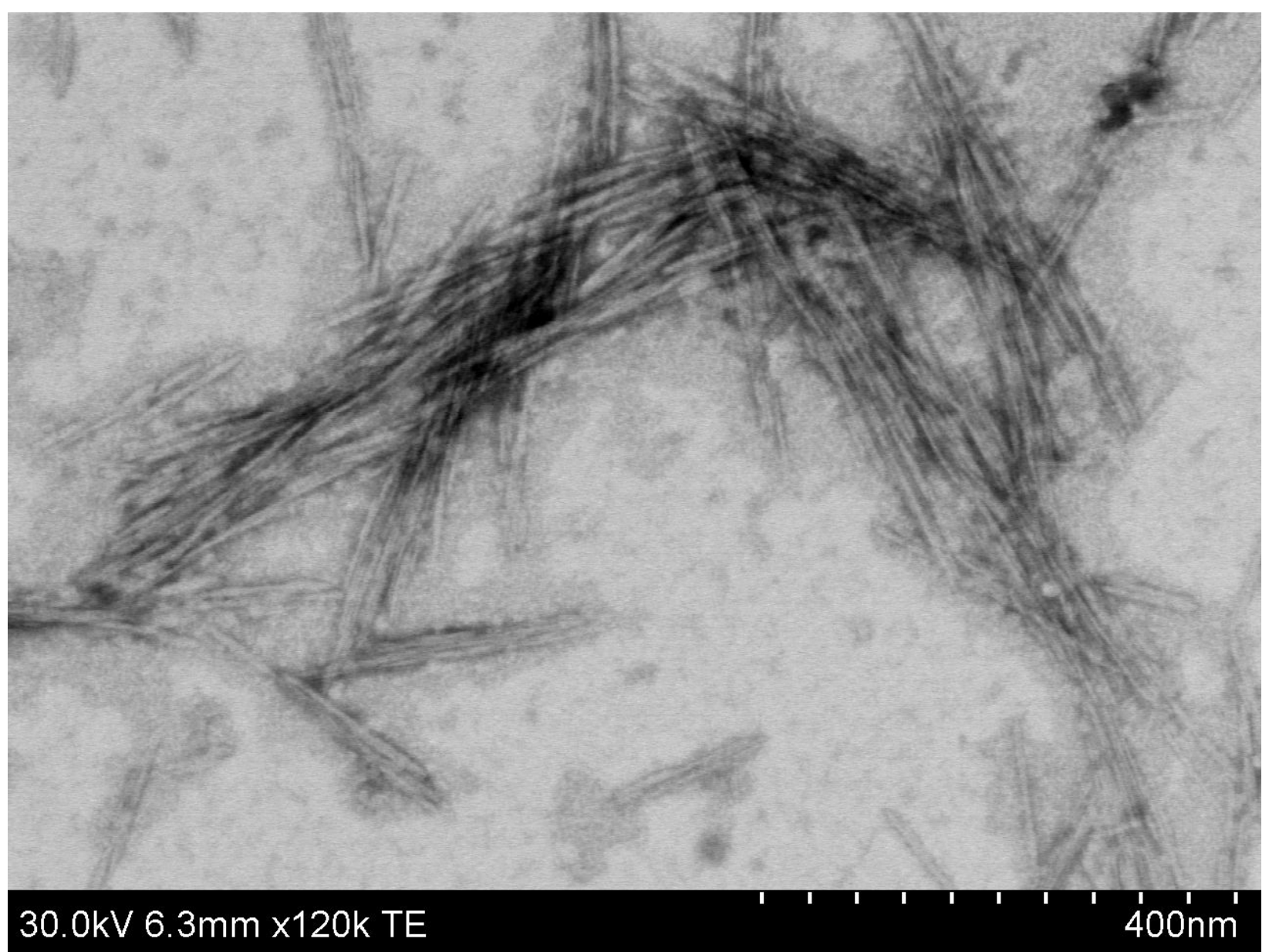




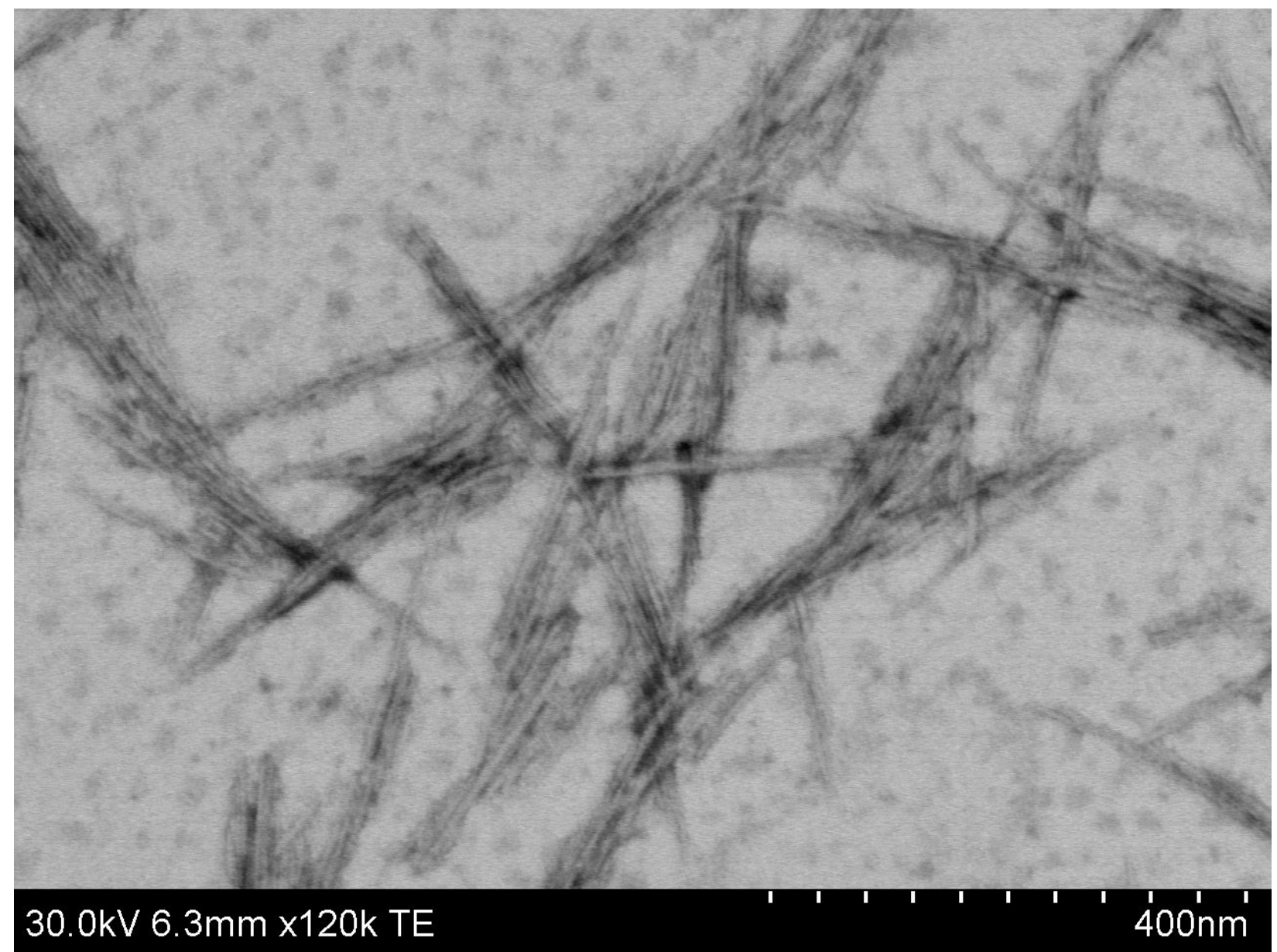

Figure S1. STEM images of $0.01 \%$ CNC suspension

\section{Isothermal titration calorimetry (ITC)}

ITC was used to investigate the adsorption of OPE-100 molecules onto CNCs surfaces at 25 and $70^{\circ} \mathrm{C}$ (Figure A2). The adsorption of OPE-100 onto CNCs is of particular relevance in the spray drying process. $\mathrm{CNC}-\mathrm{OPE}-100$ aerosol particles reach a temperature close to $86-90^{\circ} \mathrm{C}$ (outlet temperature).

At $25^{\circ} \mathrm{C}$ the heat signal of initial injections of OPE-100 $5 \mathrm{mM}$ into water account for the dissociation of injected micelles into surfactant's monomers and can be seen as a nearly constant change of enthalpy (OPE-100 concentration below $0.2 \mathrm{mM}$ ). With subsequent injections the 
concentration of OPE-100 increases and surfactant molecules start to associate into micelles (critical micellar concentration $0.29 \mathrm{mM}$ ), resulting in increased enthalpy values. After formation of micelles, only dilution of injected micelles takes place. The reference curve, which is the addition of OPE-100 into distilled water, can be considered as a dilution curve and can be classified in three regions. During the first (early) stage of titration, the large exothermic heat effects were due to complete demicellization of the injected micelles of OPE-100. Titrant OPE100 was in micellar form before injecting into water. Since the final concentration in water is below CMC, the added micelles disintegrate into OPE-100 molecules and heat was released. The second region was due to the transition from individual OPE-molecules to micelles. The third region corresponds above CMC. Titrant OPE-100 is only diluted; however, micelles remained intact. The enthalpy of demicellization was approximately $6 \mathrm{~kJ} / \mathrm{mol}$. as measured from the difference between the first and third regions. The differences between the shapes of titration curves of OPE-100 in CNC suspension and water can be described as surfactant OPE-100 and CNC particles interaction.

In the presence of CNCs, adsorption on CNC surfaces and micellization of OPE-100 is occurred at a lower concentration $(0.21 \mathrm{mM})$. Diluted monomers start to interact and adsorb to CNCs' surfaces at concentrations below $0.2 \mathrm{mM}$ as evidenced by the increasing $\Delta \mathrm{H}$ values. Subsequent injections of OPE-100 molecules result in aggregation of monomers into micelles adsorbed on CNCs and in water. A similar phenomenon is observed at $70^{\circ} \mathrm{C}$, however, in this case the micellization process is exothermic. At this temperature increased molecular motions overcome the intermolecular hydrogen bonding forces between the oxygen of the ethoxylated chain of OPE-100 and hydrogen of water molecules. Hence, surfactant molecules start to aggregate spontaneously through hydrophobic interactions at a concentration slightly below $0.2 \mathrm{mM}$. The 
aggregation and micelle formation processes are accelerated in presence of CNCs as shown in Figure S2. Although the residence time of drying droplets is extremely short, the high temperature of droplets $\left(86-90^{\circ} \mathrm{C}\right)$ above the cloud point of OPE100 $\left(66^{\circ} \mathrm{C}\right)$ accelerates the formation of larger micelles that can prevent the aggregation of CNCs during the formation of granules.
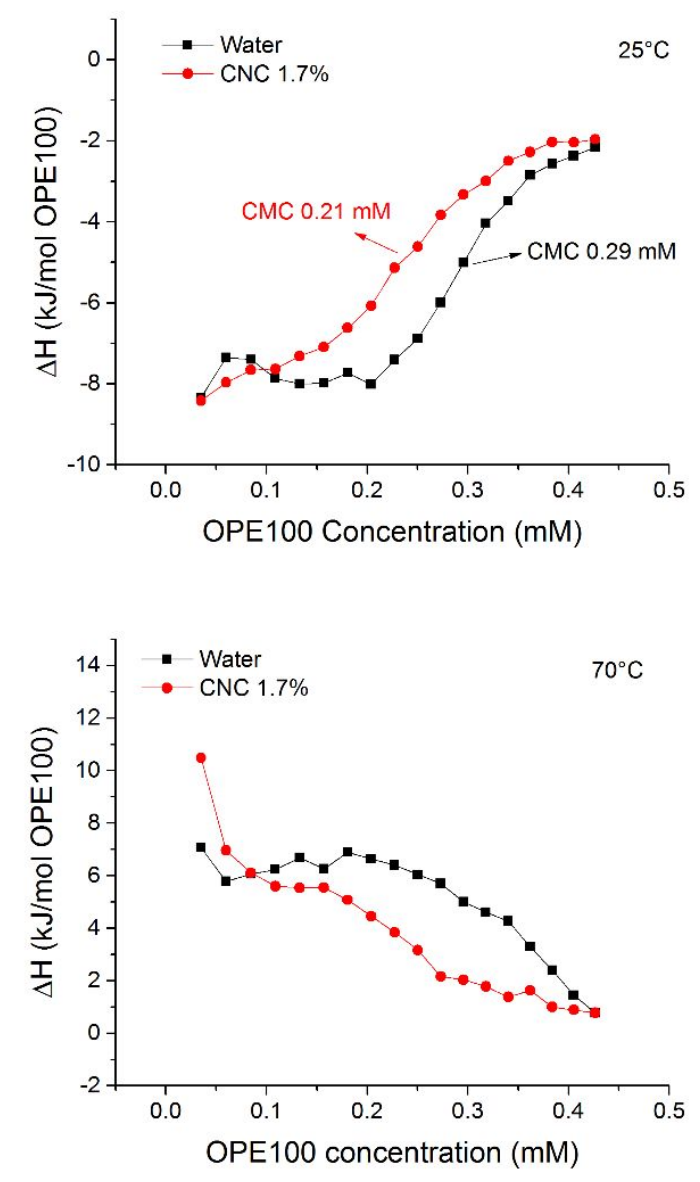

Figure S2. Isothermal titration calorimetry plots of titration of OPE-100 5 mM solution into water and $\mathrm{CNC} 1.7 \%$ at $25^{\circ} \mathrm{C}$ and $70^{\circ} \mathrm{C}$. 